\title{
BMJ
}

\section{Reduced in-hospital mortality after improved management of children under 5 years admitted to hospital with malaria: randomised trial}

\author{
Sidu Biai, director of paediatric ward, ${ }^{1}$ Amabelia Rodrigues, senior epidemiologist, ${ }^{1}$ Melba Gomes, projects \\ manager, ${ }^{2}$ Isabela Ribeiro, clinical epidemiologist, ${ }^{2}$ Morten Sodemann, clinical epidemiologist, ${ }^{1}$ \\ Fernanda Alves, clinical epidemiologist, ${ }^{3}$ Peter Aaby, professor $^{1}$
}

${ }^{1}$ Projecto de Saúde de Bandim, INDEPTH Network, Bissau Codex 1004, Guinea-Bissau

${ }^{2}$ World Health Organization, Tropical Disease Research, 1211 Geneva 27, Switzerland

${ }^{3}$ World Health Organization, Bissau Codex 1011, Guinea-Bissau Correspondence to: S Biai sidubiai@hotmail.com

doi:10.1136/bmj.39345.467813.80

\section{ABSTRACT}

Objective To test whether strict implementation of a standardised protocol for the management of malaria and provision of a financial incentive for health workers reduced mortality.

Design Randomised controlled intervention trial. Setting Paediatric ward at the national hospital in Guinea-Bissau. All children admitted to hospital with severe malaria received free drug kits.

Participants 951 children aged 3 months to 5 years admitted to hospital with a diagnosis of malaria randomised to normal or intervention wards. Interventions Before the start of the study, all personnel were trained in the use of the standardised guidelines for the management of malaria, including strict follow-up procedures. Nurses and doctors were randomised to work on intervention or control wards. Personnel in the intervention ward received a small financial incentive ( $\$ 50$ ( $£ 25 ; € 35$ )/month for nurses and $\$ 160$ for doctors) and their compliance with standard case management was closely monitored.

Main outcome measures In-hospital mortality and cumulative mortality within 4 weeks of hospital admission.

Results In-hospital mortality was $5 \%$ for the intervention group and $10 \%$ in the control group (risk ratio 0.48, 95\% confidence interval 0.29 to 0.79 ). The effect may have been stronger in patients with positive malaria slides $(0.36,0.16$ to 0.80$)$. Cumulative mortality 4 weeks after discharge was also lower in the intervention group $(0.61,0.40$ to 0.95$)$.

Conclusions Supervising healthcare workers to adhere to a standardised treatment protocol was associated with greatly reduced in-hospital mortality. Financial incentives may be important for the dedication and compliance of staff members.

Trial registration Clinical Trials NCT00465777.

\section{INTRODUCTION}

Paediatric hospital wards in developing countries have failed to respond adequately to the challenge of saving severely sick children. ${ }^{1}$ Problems have been identified in triage and emergency care, in monitoring procedures and follow-up of treatment guidelines, and in the unavailability of drugs. ${ }^{2}$ These factors have contributed to high mortality in hospitals in which health personnel are poorly paid and unmotivated. Staff training alone is not a solution, and organisational difficulties and follow-up of patients have been identified as important problems. ${ }^{2}$

Mortality on the paediatric ward of the national hospital Simao Mendes in Guinea-Bissau (northwest Africa) is high. ${ }^{3}$ It varies between $12 \%$ and $18 \%$, and malaria specific mortality was $12 \%$ in 2004. From $1991-6$, 56\% (376/672) of deaths occurred within 24 hours of admission. ${ }^{3}$ Similar patterns have been seen in studies from Kenya and Zimbabwe, where $84 \%$ and $44 \%$ of deaths occurred within 24 hours of admission. ${ }^{45}$

Community studies of childhood mortality in low income countries have highlighted the poor performance of local health services and have emphasised that future health interventions should also be directed towards hospitals. ${ }^{67}$ Effective interventions to reduce in-hospital mortality in low income countries should improve equity and reduce mortality in the community.

Paradoxically, during a civil war in 1998-9, mortality in hospital in children under 15 years decreased significantly (odds ratio $0.58,95 \%$ confidence interval 0.50 to 0.68$)$, as did mortality after discharge $(0.57$, 0.40 to 0.83$).{ }^{8}$ This was not explained by a general decline in mortality during the war, and children admitted to hospital during the war did not seem to be less sick or more advantaged socioeconomically. Mortality in the community increased by 34\% during the war. The reduction in mortality during the war may have been the result of several changes on the ward. Firstly, drugs donated by the emergency aid organisations were available free of charge; secondly, staff attendance on the ward was better, as some members of staff lived on the ward and were supported with relief food from the World Food Programme; thirdly, morale of the staff was higher and they were more dedicated.

About 70\% of children admitted to hospital have a clinical diagnosis of malaria, and around $44 \%$ of children 
under 5 have parasitologically confirmed malaria. ${ }^{9}$ These observations led the National Malaria Control Programme and the paediatric ward - in collaboration with the Bandim Health Project (BHP) and the World Health Organization (WHO) country office-to demand drug kits for emergency management of children with severe and complicated malaria. These kits were funded first by BHP and then by WHO. However, we found no clear reduction in mortality after the kits were introduced (1 October 2000 until 16 October 2003). Hence, we investigated whether adherence to existing standardised guidelines for the management of malaria and strict monitoring of the patients could reduce mortality. To make supervision and corrections more acceptable we decided to offer a small financial incentive. We evaluated in a randomised trial whether a standardised protocol for the management of malaria-including the enforcement of strict procedures for monitoring of patients, availability of free drugs, and small financial incentives - could reduce mortality on the paediatric ward.

\section{MATERIAL AND METHODS}

\section{Study site}

This study was carried out on the paediatric ward of the national hospital. This is the national referral paediatric

Children aged 3 months to 5 years admitted during the study period $(n=3122)$

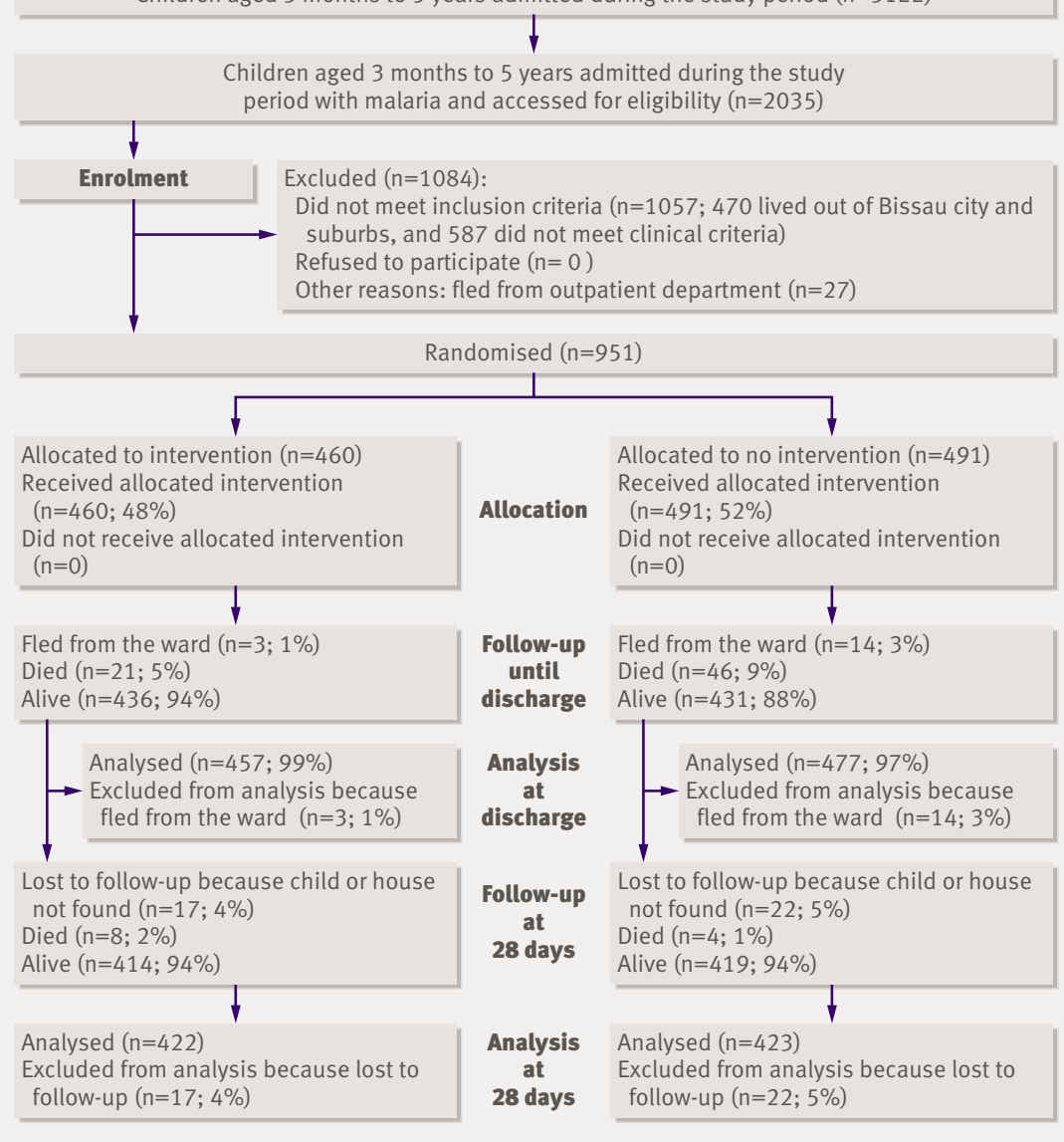

ward, but it serves also as a primary healthcare contact for children from Bissau city and suburbs. These children may go straight to the hospital without being referred through a health centre. During the study period, the paediatric ward had 117 beds and there was one doctor for each 12 beds and one nurse for each 32 beds during duty time. The 15 beds in the intensive care unit were covered by two nurses from 8 am until 2 pm and one nurse for the rest of the day. All doctors rotate in the outpatient clinic. Every morning, each doctor visits his or her patients and patients are discharged at this stage.

Standard procedure is as follows. Children arrive at the outpatient clinic and wait to see a doctor. Very sick children are screened by a nurse and sent to the doctor as soon as possible. The doctor evaluates the child and decides whether he or she should be admitted, remain under observation, or be given ambulatory treatment. The doctor might ask for a laboratory test. The consultation fee is $\$ 0.5(£ 0.25 ; € 0.35)$ and laboratory tests are charged separately; for example, a thick film, a total leucocyte count, and a haemoglobin measurement each cost $\$ 1$. The hospital stay is free of charge. After consultation, the parents are given a prescription and they have to buy the necessary drugs. Drugs can be bought from the central hospital pharmacy or the internal paediatric pharmacy when available, and if the parents can afford them. Parents may have to go back to their home to raise money. They may also have to visit a private pharmacy to buy drugs that are not available at the hospital. When admitted to hospital, children are treated with whatever drugs the parents can afford.

\section{Study design}

We conducted a randomised controlled study of the effect of a composite intervention (improved management of malaria, free emergency drugs for malaria, and financial incentive) on the hospital mortality rate. One part of the ward was designated the intervention ward and the other was designated the reference or control ward.

\section{Training of staff}

Medical staff and nursing staff from both the intervention ward and control ward were trained in the use of the standardised protocol for managing malaria.

\section{Randomisation of staff}

After training, three doctors and eight nurses were randomly selected for the intervention ward (consisting of four rooms), and the remaining personnel continued to work on the control ward (again, four rooms), which was also staffed by three doctors and eight nurses.

\section{Outpatient clinic}

In the outpatient clinic, patients consulted with the doctor in charge as usual and the doctor decided whether or not to admit the child to hospital. All children who were admitted with a clinical diagnosis of malaria and who met the criteria for enrolment were invited to 
participate in the study. The doctor completed a form containing demographic and clinical information. A laboratory technician collected thin and thick blood films, which were stained with Giemsa. The films were read on the ward and the results were given to the doctor. The slides were then sent to the BHP, where they were read again by a researcher. This second set of readings was used in the study.

\section{Enrolment and consent procedures}

As soon as the doctor decided to admit a child fulfilling the inclusion criteria, a study assistant explained the purpose of the study to the parents and asked for consent to include the child. When informed consent was obtained, the assistant randomised the child by opening a sealed envelope which contained the assignment to one of the two groups. All children admitted to the hospital with malaria, aged 3 months to 5 years and living in Bissau city and surrounding suburbs, were randomly referred to the standard ward or the intervention ward. All children admitted received the antimalarial kit free of charge. Patients temporarily transferred to the intensive care unit were transferred back to the ward to which the child was originally assigned.

\section{Inclusion criteria}

We included children aged 3 months to 5 years who had clinically suspected malaria, defined as axillary temperature $>37.5^{\circ} \mathrm{C}$ or a history of recent fever with no other obvious cause. We also required the presence of one or more of the following conditions: unable to eat, suck, or drink; more than three vomiting episodes in the previous 24 hours; unable to sit or stand; impaired consciousness (Blantyre coma score 3-4); cerebral malaria (two or more convulsions over the preceding 24 hours); coma (Blantyre coma score 0-2, at least 30 minutes after a generalised convulsion); or severe anaemia $(<50 \mathrm{~g} / \mathrm{l})$. We excluded children without the above conditions or if the parents or carer refused to give consent.

\section{Randomisation procedure and masking}

We generated 1000 randomisation numbers on the computer and assigned them a running number. Each child's randomisation number was sealed inside an

\begin{tabular}{|c|c|c|}
\hline \multirow[b]{2}{*}{ Variable } & \multicolumn{2}{|c|}{ Group } \\
\hline & Intervention & Control \\
\hline Sex (male) & $251 / 460(55)$ & $280 / 491(57)$ \\
\hline Median (interquartile range) age in months & $24(13-36)$ & $24(14-39)$ \\
\hline Arm circumference $<130 \mathrm{~mm}$ & $54 / 454(12)$ & $60 / 489(12)$ \\
\hline High transmission season & $258 / 460(56)$ & $269 / 491(55)$ \\
\hline House with ceiling & $4 / 455(0.9)$ & $12 / 489(2.5)$ \\
\hline Impregnated bed net & $77 / 455(17)$ & $89 / 488(18)$ \\
\hline Living in the BHP study area & $139 / 460(30)$ & $141 / 491(29)$ \\
\hline Malaria parasite positive slide & $318 / 460(69)$ & $338 / 491(69)$ \\
\hline
\end{tabular}

envelope and the running number written on the outside. Because the study was not blinded, the running number helped us ensure that randomisation was not violated - the running numbers on the envelope should match the sequential number in which children were enrolled. We assigned children with an odd number to the intervention group and those with an even number to the control group. The original randomisation list had 483 odd numbers and 517 even numbers. Hence, there was a slight imbalance between the two groups. For evident reasons, the study was not masked.

\section{Follow-up}

Children came to the hospital for a follow-up visit after 28 days. Children who could not attend the follow-up visit were seen by a project nurse in the community.

\section{The intervention}

People working in both wards were trained in the use of the standard protocol for management of malaria and all patients received the drug kits free of charge. The same conditions were present in both wards, which were separated by a corridor.

\section{Intervention ward}

All staff working on the intervention ward rigorously followed the procedures recommended in the national standardised guidelines on the management of malaria and filled in the case record forms; the doctors evaluated the nurses' quality of work and the study supervisor systematically controlled the quality of implementation of the recommended procedures. Results of supervision were registered in the form. Nurses and doctors received a small amount of money ( $\$ 50 /$ month for nurses and $\$ 160$ for doctors) for the additional forms they had to fill out. This is roughly what they would earn if they did extra part time work and is equivalent to monthly rent in Bissau for these categories of staff.

Children assigned to the intervention ward were re-evaluated by the study doctor on duty until $10 \mathrm{pm}$. The nurse on duty called one of the study doctors if a seriously sick child was recruited after $10 \mathrm{pm}$. The nurses on this ward could make management decisions themselves when the study doctor was not present.

\section{Control ward}

In the control ward, procedures were the same as usual. The national guidelines on the management of malaria were supposed to be followed. However, the lack of supervision meant that people could choose whether or not to follow the recommendations. The doctors were not obliged to evaluate the nurses' work, and the doctors themselves were not evaluated either.

\section{Main outcomes}

The primary end point was in-hospital mortality. The secondary end points were cumulative mortality after discharge at day 28 and length of hospital stay. 


\section{Content of kit}

The national malaria policy for treating severe malaria recommends intravenous infusion of $20 \mathrm{mg}$ quinine/ $\mathrm{kg}$ body weight in $5 \%$ saline solution $(4-10 \mathrm{ml} / \mathrm{kg})$ for four hours. After that, $10 \mathrm{mg}$ quinine $/ \mathrm{kg}$ body weight should be given for four hours and repeated every eight hours until the patient can be treated orally. Thus, the basic kit for severe malaria included two $500 \mathrm{ml} \mathrm{bags}$ of $5 \%$ glucose, one vial of $600 \mathrm{mg}$ of quinine, two intravenous catheters, five $5 \mathrm{ml}$ syringes, two pairs of surgical gloves, paracetamol and drugs for other conditions including one vial of diazepam, three sachets of oral rehydration salt, two $500 \mathrm{ml}$ bottles of ringer lactates, and nasogastric tubes. If the child needed other drugs to complete the treatment, the parents had to buy them.

\section{Sample size calculation}

We estimated that 474 children were needed in each arm to observe-with $80 \%$ power and a $5 \%$ significance level-a 50\% reduction in mortality (from around $10 \%$ in the control group to $5 \%$ in the intervention group).

\section{Statistical methods}

We used Dbase-VI and Stata- 8 for data entry and cleaning. Statistical analyses were carried out with Stata-8. We calculated risk ratios for in-hospital mortality and mortality after discharge. The length of hospital stay in days was compared for the two groups. Children who fled from the hospital were excluded in the analysis of in-hospital case fatality; however, if we obtained information from them at the 28 day followup they were included in the analysis of mortality after discharge. Although most of the children absconding from the ward were alive at the 28 day visit, we also conducted an analysis considering lost to follow-up at the hospital as a negative outcome. We compared the mean duration of stay on the ward and time to

\begin{tabular}{|c|c|c|c|c|}
\hline \multirow[b]{2}{*}{ Variable } & \multicolumn{2}{|c|}{ Group } & \multirow[b]{2}{*}{ Risk ratio $(95 \% \mathrm{Cl})$} & \multirow[b]{2}{*}{ Pvalue } \\
\hline & Intervention & Control & & \\
\hline Mortality at discharge & $21 / 457(5)$ & $46 / 477(10)$ & 0.48 (0.29 to 0.79$)$ & 0.002 \\
\hline \multicolumn{5}{|l|}{ In-hospital mortality } \\
\hline Patients from the BHP area & $5 / 138(4)$ & $9 / 136(7)$ & 0.55 (0.19 to 1.59$)$ & 0.26 \\
\hline $\begin{array}{l}\text { Patients living outside the } \\
\text { BHP area }\end{array}$ & $16 / 319(5)$ & $37 / 341(11)$ & $0.46(0.26$ to 0.81$)$ & 0.005 \\
\hline $\begin{array}{l}\text { Patients positive for malaria } \\
\text { on microscopy }\end{array}$ & $8 / 316(3)$ & $23 / 329(7)$ & $0.36(0.16$ to 0.80$)$ & 0.008 \\
\hline $\begin{array}{l}\text { Patients negative for malaria } \\
\text { on microscopy }\end{array}$ & $13 / 141(9)$ & $23 / 148(16)$ & 0.59 (0.31 to 1.12$)$ & 0.10 \\
\hline \multicolumn{5}{|l|}{ Mortality after 28 days } \\
\hline Cumulative & $29 / 443(7)$ & $50 / 469(11)$ & 0.61 (0.40 to 0.95$)$ & 0.02 \\
\hline $\begin{array}{l}\text { Patients positive for malaria } \\
\text { on microscopy }\end{array}$ & $12 / 307(4)$ & $26 / 324(8)$ & $0.49(0.25$ to 0.95$)$ & 0.02 \\
\hline $\begin{array}{l}\text { Patients negative for malaria } \\
\text { on microscopy }\end{array}$ & $17 / 136(13)$ & $24 / 145(17)$ & $0.76(0.42$ to 1.34$)$ & 0.33 \\
\hline
\end{tabular}

discharge between the two groups using Cox regression.

To compare mortality for the same age group before the trial we compared mortality during the year of the trial with mortality between December 2003 and November 2004-the 12 months before the start of the trial.

\section{Ethics}

All children enrolled in the study should have benefited. The children in the control and research wards benefited from the free drug kits and the retraining of the staff. The patients did not pay the fees for laboratory tests as the BHP provided the materials and reagents. The children in the research ward would also have benefited from the personnel being rigorously supervised. A data safety monitoring board has not been established for financial reasons.

\section{RESULTS}

The trial was conducted between 1 December 2004 and 16 January 2006. During this time, 3122 children between 3 months and 5 years were admitted to the hospital. Among them, 2035 were clinically diagnosed with malaria; 470 lived outside Bissau city, 587 did not meet the inclusion criteria, and 27 fled before randomisation. We randomised 951 children: $460(48 \%)$ to the intervention group and $491(52 \%)$ to the control group. At the hospital, three $(0.7 \%)$ children fled from the intervention group (two seen alive at 28 days, one lost to follow-up), while 14 (3\%) fled from the control group (12 seen alive at 28 days, two lost to follow-up) (fig 1). In the whole group the diagnosis was revised to severe anaemia in 172 children, bronchopneumonia in 78 patients, diarrhoea in 32, typhoid fever in nine, and sepsis in four. Of the 295 patients with a negative malaria slide, we diagnosed severe anaemia in 45 , bronchopneumonia in 26 , diarrhoea in 17 , sepsis in two, and typhoid fever in one.

Background characteristics were similar in the two groups: $251 / 460(55 \%)$ were boys in the intervention group and 280/491 (57\%) in the control group. The median age of children admitted was 24 months in both groups. Randomisation to the groups was similar for different transmission seasons, and the proportion of patients with positive malaria slides was the same $(69 \%)$ in both groups. Socioeconomic conditions were comparable and the same proportion of children used impregnated bed nets (table 1).

In the 12 months before the trial, overall mortality at the hospital in children aged between 3 months and 5 years was $13 \%(413 / 3076)$. During the period of the study from December 2004 to January 2006, 3122 children were admitted to the hospital; no information on outcome was available for 331 children who left without discharge. Of the remaining 2791 children, mortality was $13 \%(351 / 2791)$. 
In-hospital mortality

We excluded the 17 children who fled from the analysis of in-hospital mortality. All patients included in the trial were clinically diagnosed with malaria. Of the 67 children who died in hospital, 36 had a diagnosis of malaria only, 20 had anaemia as a secondary diagnosis, six had pneumonia, four had diarrhoea, and one had convulsions. In the intervention group, $21 / 457(5 \%)$ patients died and in the control group $46 / 477(10 \%)$ patients died (risk ratio 0.48, 95\% confidence interval 0.29 to $0.79, \mathrm{P}=0.002$ ). The effect was similar in boys $(10 / 251(4 \%)$ v 26/270 (10\%); 0.41, 0.20 to 0.84$)$ and girls $(11 / 206(5 \%)$ v 20/207 (10\%); $0.55,0.27$ to 1.12 ). Children living in or outside the BHP study area had a similar reduction in mortality, although mortality was lower for children from the study area (table 2).

Mortality was significantly higher in children without confirmed malaria $(12 \%)$ than in those clinically diagnosed with malaria $(5 \%)(\mathrm{P}=0.04$; table 2$)$. Because the inclusion criteria were based on a clinical diagnosis of malaria, we looked at the effect of intervention on the basis of whether malaria was confirmed by microscopy or not. Most of the effect of the intervention was seen in children who had malaria positive slides $(0.36$, 0.16 to 0.80 ).

Fewer patients absconded in the control group than in the intervention group $(3 / 460(0.7 \%)$ v $14 / 491$ $(2.9 \%) ; 0.23,0.07$ to 0.79$)$. If lost to follow-up at the hospital was included as a negative outcome, the risk ratio for a negative outcome was 0.43 (0.27 to 0.67 ).

\section{Length of hospital stay}

The mean length of stay on the paediatric ward was shorter in the intervention group ( 7 days, standard deviation 3.2) than in the control group (8 days, 4.2), as the rate of discharge was $35 \%$ shorter in the intervention group $(18 \%$ to $55 \%, \mathrm{P}<0.0001)$.

\section{Mortality at $\mathbf{2 8}$ days of follow-up}

At the 28 day visit, we obtained information on 422 children in the intervention group -17 were lost to follow-up. In the control group, we obtained information on 423 children-22 were lost to follow-up. Children from the intervention group were twice as likely to come for the 28 days visit at the hospital than children from the control group $(55 \% v 25 \% ; 2.15,1.79$ to $2.59, \mathrm{P}<0.0001)$.

When we looked at mortality after discharge alone, the intervention group had slightly higher mortality than the control group $(2.01,0.61$ to $6.64, \mathrm{P}=0.23)$. However, overall mortality at day 28 was lower in the intervention group than in controls $(7 \%$ v 11\%; 0.61 , 0.40 to 0.95$)$. The effect was strongest for the laboratory confirmed malaria cases $(4 \% v 8 \% ; 0.49,0.25$ to 0.95 ; table 2 ).

\section{DISCUSSION}

We found that a composite intervention-including standardised guidelines on the management of malaria, free emergency drugs for malaria, and modest financial incentives to staff-reduced in-hospital mortality for patients with malaria by half. Because all staff were trained before the study and randomised to the intervention or control wards, and all children received free emergency drugs, neither training nor free drugs alone affected the quality of management and subsequent survival.

The inclusion criteria for patients with malaria were based on clinical diagnosis, as is usually the case in low income countries. The intervention tended to be more beneficial in patients with confirmed malaria. This suggests that laboratory tests should be used when deciding whether or not to treat patients with antimalarial drugs. The tendency to overdiagnose malaria may mean that less attention is paid to other serious conditions. This could contribute to the high mortality seen in such patients. ${ }^{1011}$

The study aimed to reduce in-hospital mortality, and the health staff could have discharged children earlier to avoid deaths in hospital. Children in the intervention group had a shorter median length of hospital stay than those in the control group, yet they had lower cumulative mortality after one month. The extra care and attention given to the patients seemed to influence how well they adhered to the advice of health personnel and increased their confidence in the health system, as twice as many children in the intervention group came to the hospital for the follow-up visit.

Our study could not distinguish between the effect of supervising the implementation of guidelines and the effect of the financial incentive in reducing mortality. However, it would be difficult to separate these factors in our setting. To make the staff accept corrections and changes in behaviour after supervision, they need to be interested and available, otherwise neither the supervision nor the training would have any effect.

Both the intervention and the control wards were situated in the same building and the staff communicated with each other. There could have been contamination between the staff, from the two wards. However, this would have reduced the estimated effect.

Training is one of the main activities of health programmes, but it should be followed up by support and reinforcement. The same applies to the availability of free drugs. With the cost recovery system that is normally in place patients also pay for urgent drugs, and parents often need to go home to raise the money. Having to pay for services and drugs in health facilities might make certain interventions less effective. ${ }^{12}$ Free drugs are an important part of our intervention, but drugs alone are not likely to have a large effect on mortality if the patient's care is not good. In resource constrained settings, hospital workers earn very little, payment is often delayed, and they are not highly motivated to improve performance. Supervision is lacking or ineffective. Even a small financial incentive and a specific target (such as mortality) can make workers more likely to adhere to standards and improve the quality of their work. Targets can divert effort away from other activities,${ }^{13}$ but mortality is the 


\section{WHAT IS ALREADY KNOWN ON THIS TOPIC}

Health care in African hospitals is of low quality

Health personnel in African hospitals are poorly motivated

\section{WHAT THIS STUDY ADDS}

In poor countries, small financial incentives can contribute to a dramatic decrease in

in-hospital mortality

Training and free drugs alone are not enough to reduce mortality outcome of several interventions, and any intervention undertaken in the paediatric wards that reduces mortality would contribute to better quality of care. In our setting, training seminars and other activities within new global initiatives provide workers with extra payments and subsidies, but most of these activities distract the scarce staff from their real job.

It is unclear whether financial incentives improve quality of care in developed countries. ${ }^{1314}$ However, in developing countries, most healthcare personnel do other work to supplement their income. ${ }^{15-17}$ Indeed, many can only stay in the health sector if they can generate extra income. Hence, interventions that provide financial incentives to staff in poor countries are likely to have more effect than those in developed countries.

\section{Conclusions}

We have shown that quality of care in the paediatric ward, based on impact indicators, depends not only on training and availability of drugs, but also on financial incentives. Our results are probably relevant to other national and district hospitals in developing countries that face the same conditions and problems -namely, low quality of care and staff with little motivation. ${ }^{118}$ Attempts to motivate staff in primary healthcare settings might also be beneficial. Half of the deaths caused by malaria in the hospital could be prevented, and effective interventions on the paediatric ward could influence mortality in the community. ${ }^{6}$ Translating these results into policyparticularly giving financial incentives - is not easy as most donors refuse to top-up salaries. Our results imply that consideration should be given to supporting health staff salaries, possibly on the basis of performance targets. In addition, in the emergency setting, health care should not depend on prompt payment from the patients. Urgent interventions and drugs should not depend on payments from the patients or their families. Other mechanisms for compensation should be created.
Contributors: SB, AR, MG, IR, and PA planned the study. SB and AR conducted the study. MS established the BHP hospital based system. FA implemented and supervised the drug kit. SB, AR, and PA did the analysis. $\mathrm{SB}$ and $\mathrm{AR}$ wrote the first draft of the paper and all authors contributed to the final version of the paper

Funding: Not externally funded

Competing interests: None declared.

Ethical approval: National Ethical Committee "Núcleo de Coordenação das pesquisas".

1 Nolan T, Angos P, Cunha AJ, Muhe L, Qazi S, Simoes EA, et al. Quality of hospital care for seriously ill children in less-developed countries. Lancet 2001;357:106-10.

2 Zucker JR, Lackritz EM, Ruebush TK, Hightower AW, Adungosi JE, Were JB, et al. Childhood mortality during and after hospitalization in western Kenya: effect of malaria treatment regimens. Am / Trop Med Hyg 1996;55:655-60.

3 Veirum JE, Sodemann M, Biai S, Jensen H, Juul S, Aaby P. Increased mortality among children discharged from a paediatric ward in Bissau, Guinea Bissau, 1991-97. Acta Paediatr (in press).

4 Menge I, Esamai F, Van Reken D, Anabwani G. Paediatric morbidity and mortality at the Eldoret district hospital, Kenya. East Afr Med 1995;72:165-9.

5 Chawla V, Haufton B. Pattern of childhood mortality at Harare central hospital, Zimbabwe. East Afr Med / 1988;65:238-45.

6 Sodemann M, Jakobsen MS, Molbak K, Alvarenga IC, Aaby P. High mortality despite good care-seeking behaviour: a community study of childhood deaths in Guinea-Bissau. Bull World Health Organ 1997;75:205-12.

7 Reyes H, Perez-Cuevas R, Salmeron J, Tome P, Guiscafre H, Gutierrez G. Infant mortality due to acute respiratory infections: the influence of primary care processes. Health Policy Plan 1997;12:214-23.

8 Sodemann M, Veirum J, Biai S, Nielsen J, Bale C, Jakobsen MS, et al. Reduced case fatality among hospitalised children during a war in Guinea-Bissau: a lesson in equity. Acta Paediatr 2004;93:959-64.

9 Rodrigues A, Schellenberg JA, Kofoed PE, Aaby P, Greenwood B. The changing pattern of malaria in Bissau, Guinea-Bissau. Trop Med Int Health (in press).

10 Nabiswa AK, Makokha JD, Godfrey RC, Lore W. Management of malaria before and after introduction of a treatment protocol at the Eldoret district hospital. East Afr Med J 1994;71:9-13.

11 Reyburn H, Mbatia R, Drakeley C, Carneiro I, Mwakasungula E, Mwerinde 0 , et al. Overdiagnosis of malaria in patients with severe febrile illness in Tanzania: a prospective study. BMJ 2004;329:1212.

12 Victora CG, Schellenberg JA, Gwatkin D, Claeson M, Habicht JP. Applying an equity lens to child health and mortality: more of the same is not enough. Lancet 2003;362:233-41.

13 Chaix-Couturier C, Durand-Zaleski I, Jolly D, Durieux P. Effects of financial incentives on medical practice: results from a systematic review of the literature and methodological issues. Int I Qual Health Care 2000;12:133-42.

14 Petersen LA, Woodard LD, Urech T, Daw C, Sookanan S. Does pay-for-performance improve the quality of health care? Ann Intern Med 2006;145:265-72.

15 Roenen C, Ferrinho P, Van Dormael M, Conceição MC, Van Lerberghe W. How African doctors make ends meet: an exploration. Trop Med Int Health 1997;2:127-35.

16 Ferrinho P, Van Lerberghe W, Fronteira I, Hipolito F, Biscaia A. Dual practice in the health sector: review of the evidence. Hum Resour Health 2004;2:14.

17 Jan S, Bian Y, Jumpa M, Meng Q, Nyazema N, Prakomgsai P, et al. Dual job holding by public sector health professionals in highly resource-constrained settings: problem or solution? Bull World Health Organ 2005;83:771-6.

18 Van Lerberghe W, de Béthune X, De Brouwere V. Hospitals in sub-Saharan Africa: why we need more of what does not work as it should. Trop Med Int Health 1997;8:799-808.

Accepted: 17 September 2007 